\title{
TILE SETTING ROBOT FOR EXTERIOR WALL
}

\author{
Hitoshi Tomaru'), Hitoshi Haino ${ }^{2)}$, Seiichirou Ishikawa ${ }^{3)}$
}

1) Mechatronics Research Center, Komatsu Ltd. 1200,Manda, Hiratsuka, Kanagawa, 254, Japan

2) Technical Research Institute, Hazama Corporation, 4-17-23, Nishiyono, Saitama, 338, Japan

3) Japan Ceramic Tile Association, 39-18, Daikancyoh, Higashi-ku, Nagoya, 461, Japan

\begin{abstract}
Komatsu Ltd., Japan Ceramic Tile Association, and Hazama corporation organized the developing committee of construction robot in 1988, and have been working on the tile setting robot jointly. The tile setting robot has two functions, one is to set tile for exterior wall of building, and the other is to lay (mortaring and finishing) undercoat of building.

In this paper, we discuss the outline of the robot, and the results of indoor model wall test to verify functions and effectiveness of the robot, and the results of experiment applied for the construction work.
\end{abstract}

\section{Introduction}

In recent years, the shortage of craftsman, particularly young, has been more and more serious problem in the industry of construction.

In these situations, many attempts to develop the robot for construction field, because the robot contributes for the the demands for rationalization and higher productivity in the construction work. In the work of tile setting, the same problem is also serious, because the demands for ceramic tile walls increase for its decorative effect in spite of the shortage of craftsman. In order to cover up the shortage of craftsman, Komatsu Ltd., Japan Ceramic Tile Association and Hazama corporation jointly have been developing the tile setting robot, to stable the quality of execution and to improve the productivity. And now, we have proved its effectiveness by introducing the tile setting robot into the construction work.

In this paper, we describe the outline of the tile setting robot, and the results of experiment.

\section{Developing process}

Our joint development consists of three phases as followings. The first phase is to investigate the basic concept and the system of working by the robot for tile setting, and to verify the possibility of robotization by some elementary experiments. The second phase, which is described in this paper mainly, is to design and to make prototype robot, and afterward, to verify the performance and the effectiveness of the robot by examining indoor model wall test and field test. And in the last phase, we will go forward to the advanced robot which is able to be commercialized.

\section{Outline of the robot}

In this section, outline of the robot is described, that is, basic concept and main specifications of the robot.

3.1 Basic concept of the robot

Before designed the tile setting robot, following basic concept was investigated. 
The robot has two functions. One is to lay undercoat, because the bed accuracy is directly reflected in final result. The other is to set tiles. Only by changing the working unit of the robot which are laying undercoat unit and tile setting unit, the robot is able to execute these two works.

(2) Circumstance

The robot is installed on the guide rail between building and scaffolding without removing scaffolding.

(3) Movement

The robot moves on the guide rail installed on the wall of buildings after comparing with any methods, for example, hanging, adsorbing. Because, it is easy to set up the robot on the rail, and to obtain accurate undercoat and final result of tile setting.

(4) Quality

The robot achieves accurate undercoat and final tile setting surface $( \pm 2.0 \mathrm{~mm}$ tolerance for a length of $2 \mathrm{~m}$ ), and achieves good adhesive strength of undercoat and tiles ( over 6.0 $\mathrm{kgf} / \mathrm{cm}^{2}$ after wonking 28 days past) which are also better than conventional.

\subsection{Specifications of the robot}

The robot is able to execute two works, laying undercoat and setting tiles, with only changing each working unit of the robot. Outline of the robot is shown in Fig.1 and Fig. 2 .

Details of the robot are described as following.

(1) Main frame

The dimensions, of the robot is about $3.5 \mathrm{~m}$ high, width of $3.0 \mathrm{~m}$ and thickness of $0.3 \mathrm{~m}$. And weight of the robot is about $280 \mathrm{Kgf}$. The robot has two legs with spring to absorbing unevenness of the guide rail. The rail gripping units are equipped on four legs of the robot.

(2) $X$ and $Y$ slide unit

$\mathrm{X}$ and $\mathrm{Y}$ slide unit are guided by ball screw, and driven by D.C servo-motor with an optical encoder. Therefore, the obtained positioning accuracy of the robot is $0.05 \mathrm{~mm}$.

(3) Working slide unit

The working slide unit is also driven by D.C servo-motor with an optical encoder, but guided by rubber belt.

(4) Working units

Two working units for two functions as mentioned before, are explained below. They are equipped on the working slide unit according to each work.

(i) Setting tile unit

The setting tile unit has an electric power vibrator causing tiles to set firmly on undercoat laid by the robot, and a vacuum suction shocker to grasp tile. And tiles are set by the air actuator with potentiometer to measure the finishing thickness of setting mortar. (ii)Laying undercoat unit

Width of the trowel is $220 \mathrm{~mm}$, and equipped on ultrasonic sensor to measure the distance between the robot and wall. The trowel is able to move toward the wall and backward the wall, driven by D.C servo-motor with an optical encoder and a reduction gear unit.

(5) Tile supply unit

The robot sets tiles from the tile storage unit to the tile holding unit automatically. The tile storage unit can storage 160 pieces (length $108 \mathrm{~mm} *$ width $60 \mathrm{~mm} *$ thickness within $10 \mathrm{~mm}$ ), and the robot has two storage units equipped on both side of the main frame. Two tiles are adsorbed by two vacuum suction shockers which are equipped on the tile traveling unit, which carried tiles to the turning over unit. And tile is changed its posture in the turning over unit one by one. And the tile holding unit which is equipped on X and $\mathrm{Y}$ slide unit moves toward the outlet of the tile turning over unit, then the tile was set in the tile holding unit owing to be pushed by the tile pushing rod which is equipped in the turning over unit. 
(6) Tile holding unit

The tile holding unit can storage 27 pieces of tiles $(108 \mathrm{~mm} * 60 \mathrm{~mm})$, and tile can be inserted in the tile holding unit from both sides of the holder. And because the width of the holder is assembled highly accurate, the thickness of setting mortar is limited $5 \mathrm{~mm}$.

(6) Setting mortar laying unit

The setting mortar laying unit consists of the mortar storage tank and the trowel. The setting mortar storage tank is pressed by a jack with D.C servo-motor. And the trowel is driven by stepping motor. Setting mortar is pumped from the tank to the trowel through mortar hose. The pressure sensor is equipped on the trowel to detect that setting mortar is filled up or not in the trowel, and after filling up mortar, the trowel starts to lay mortar on tiles automatically.

(7) Control unit

The control unit consists of C.P.U., interface unit, power source, relays, servo amplifier units, and operating box. 32 bits computer is used for C.P.U., because C.P.U. controls many freedoms (actuators) of the robot at the same time. And as considering the electric noisy environment on site, power unit has many filtering components and safety logical circuits for fail safe.

(8) Guide rail

One piece length of the guide rail is $5 \mathrm{~m}$ and the supported span on the wall is $2.5 \mathrm{~m}$.

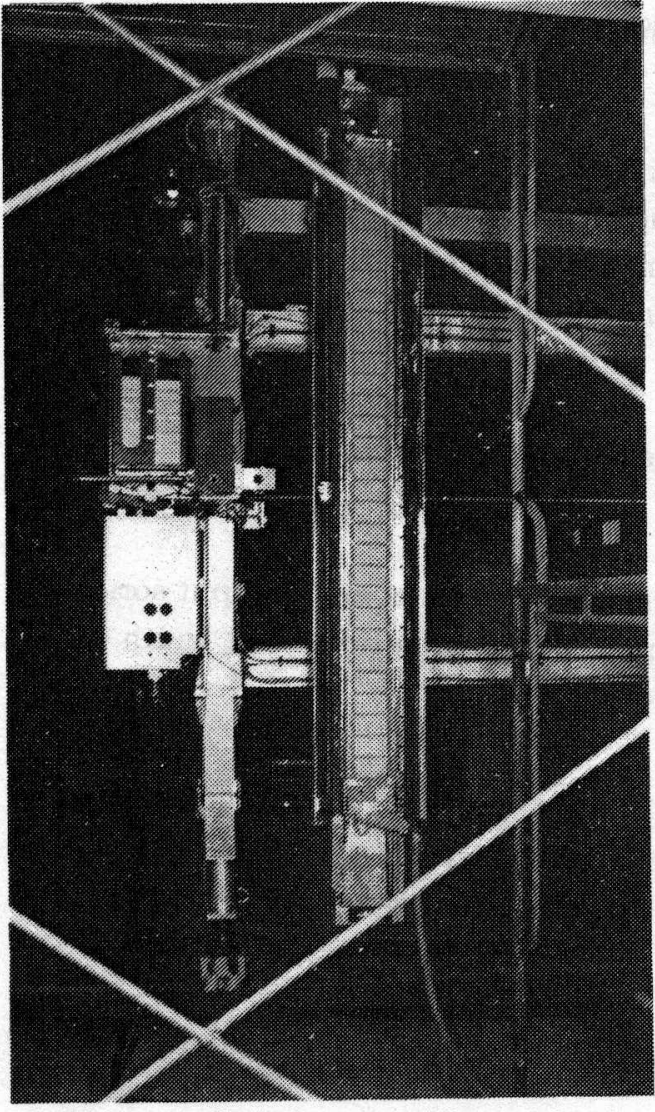

(view from wall)

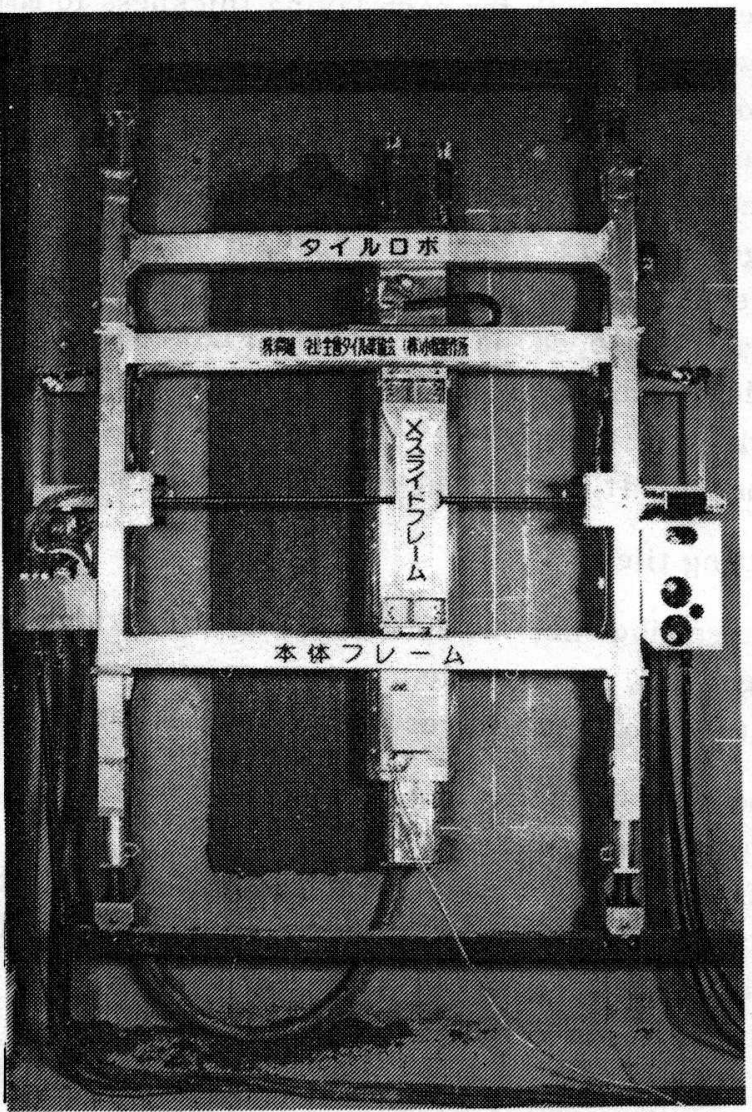

(view to wall)

Fig.1 Outlook of the tile setting robot 


\section{Results of model wall test}

Each function of the robot is tested on the indoor model wall (height $4.5 \mathrm{~m} *$ width $8 \mathrm{~m}$ ). At this time, evenness accuracy and adhesive strength of laying undercoat and setting tiles were tested. Furthermore, operating procedures for laying undercoat and setting tiles were settled on.

\subsection{Laying undercoat}

The robot is able to lay undercoat automatically corresponding to the command by only one operator. Evenness accuracy and adhesive strength were tested, and procedure of laying undercoat by the robot was settled on by the test at the same time. The results are as following.

(1) Laying procedure

(a) Teaching

By the taught information of start and end point to the robot, the robot calculates number of laying lines, acceleration and deceleration speed, and laying length. And operator teaches the laying thickness to the robot.

(b) Laying mortar

After operator pushes the start switch on the control box, the mortar supply unit starts to pump the mortar to the trowel through mortar hose automatically. And at the time of filling mortar in the trowel, the robot starts to lay mortar on with trowel upward on the taught area.

(c) Smoothing

The trowel makes smooth the rapping parts of lines without pumpimg mortar, after complete to lay all the taught area.

(2) Adhesive strength

The robot laid mortar on the test pieces which were set on the indoor model wall. Data of adhesive strength for each laying thickness $10 \mathrm{~mm}$ and $17 \mathrm{~mm}$ was obtained. The test result is shown in Fig. 3. Data shows that more strength than $6 \mathrm{Kgf} / \mathrm{cm}^{2}$ and break at itself under both conditions in break test.

(3) Evenness accuracy

The robot achieved the better evenness accuracy within $\pm 1 \mathrm{~mm}$ to a length of $2 \mathrm{~m}$ than target evenness accuracy $\pm 2 \mathrm{~mm}$ in all the test conditions. In this test, the trowel varied its speed according to measuring the distance between the robot and the wall by ultrasonic sensor equipped on the robot. Fig. 4 shows its principle, and the appearance of laying undercoat by the robot is shown in Fig. 5 .

(4) Working efficiency

Working efficiency was verified $32 \mathrm{~m}^{2} /$ day, and better than conventional.

\subsection{Setting tiles}

Onto setting tiles, no special skills for setting tiles is needed. Robot operator input some data for execution, for example, starting point to set tile, number of tiles in a line, joint width, types of joint and so. The test results of setting tiles are as following.

(1) Procedure of tile setting

(a) Teaching work area

Operator teaches tile setting area to the robot, that is, starting point to set tiles and number of tiles in a line and number of setting lines. Furthermore, type of joint, joint width. The robot is able to set cross and square joint.

(b) Setting tiles to the holder

Tiles in the tile storage unit are travelled and set to the tile holding unit automatically by the tile supply unit.

(c) Laying setting mortar on tiles

After setting all tiles to the tile holding unit, setting mortar is pumped to the trowel from the tank. And at the same time, filling up mortar in the trowel by sensing with pressure 


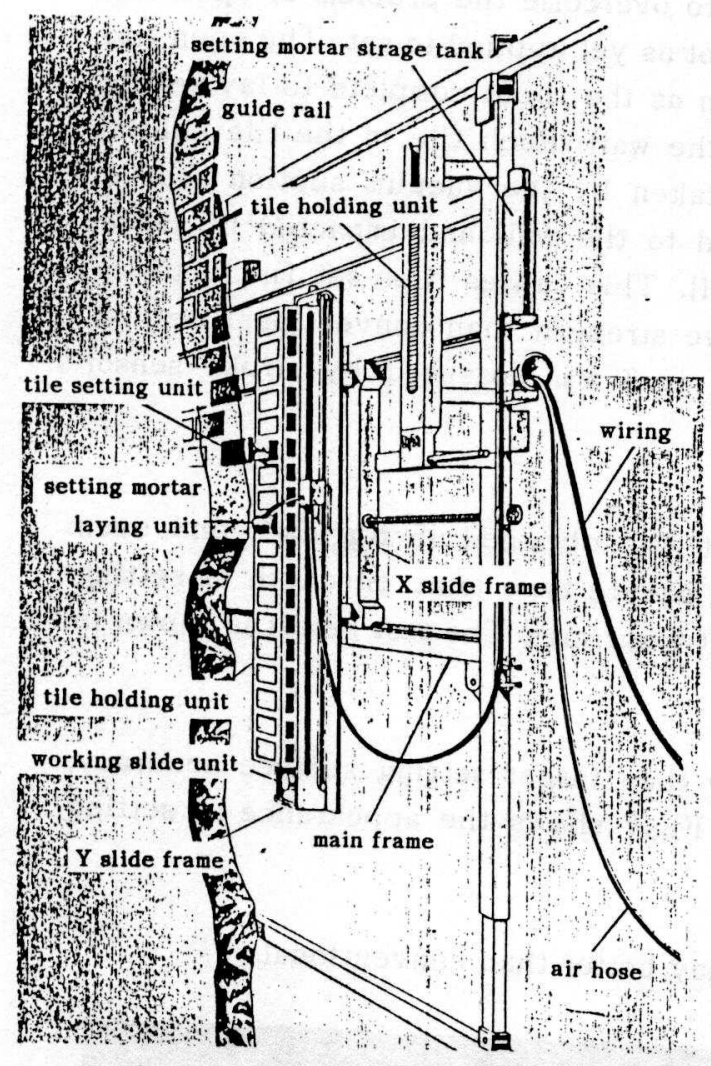

Fig.2 Outlook of the tile setting robot

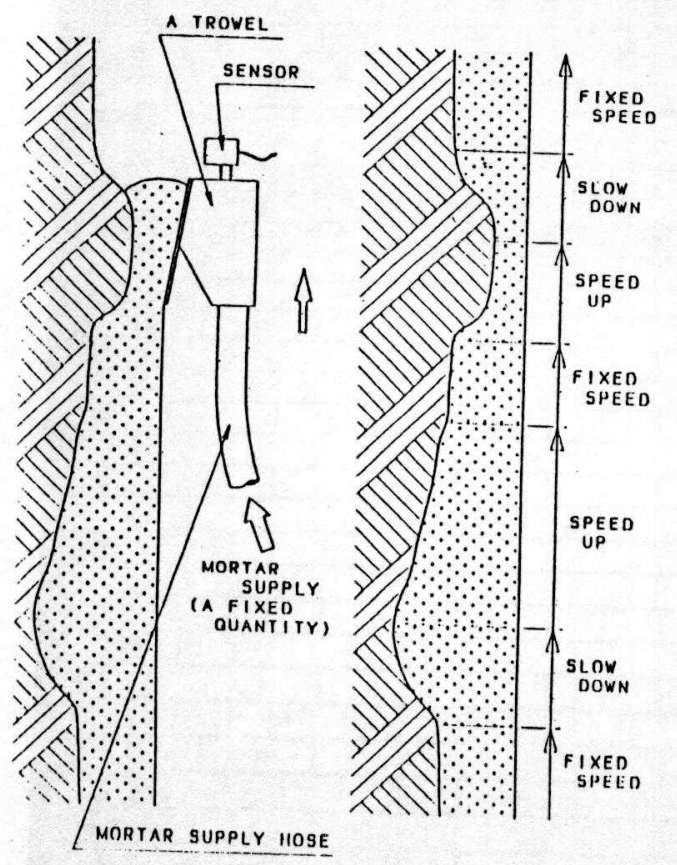

Fig.4 Trowel speed - distance between robot and wall

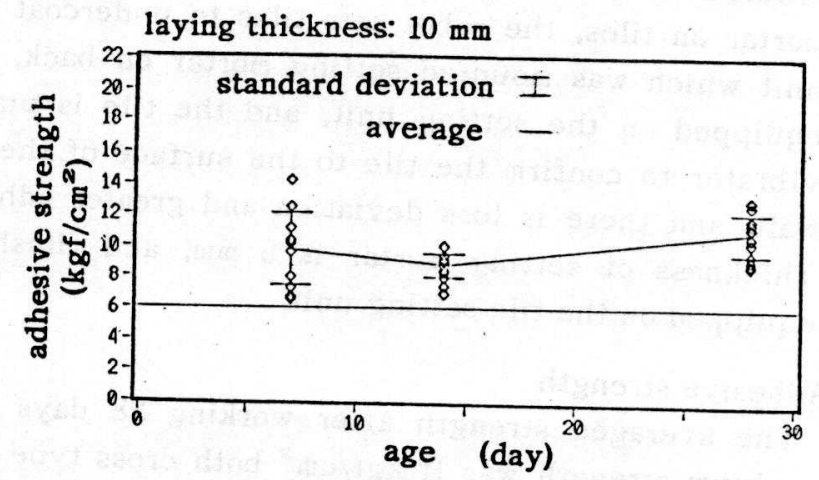

Fig. 3 Adhesive strength of laying undercoat
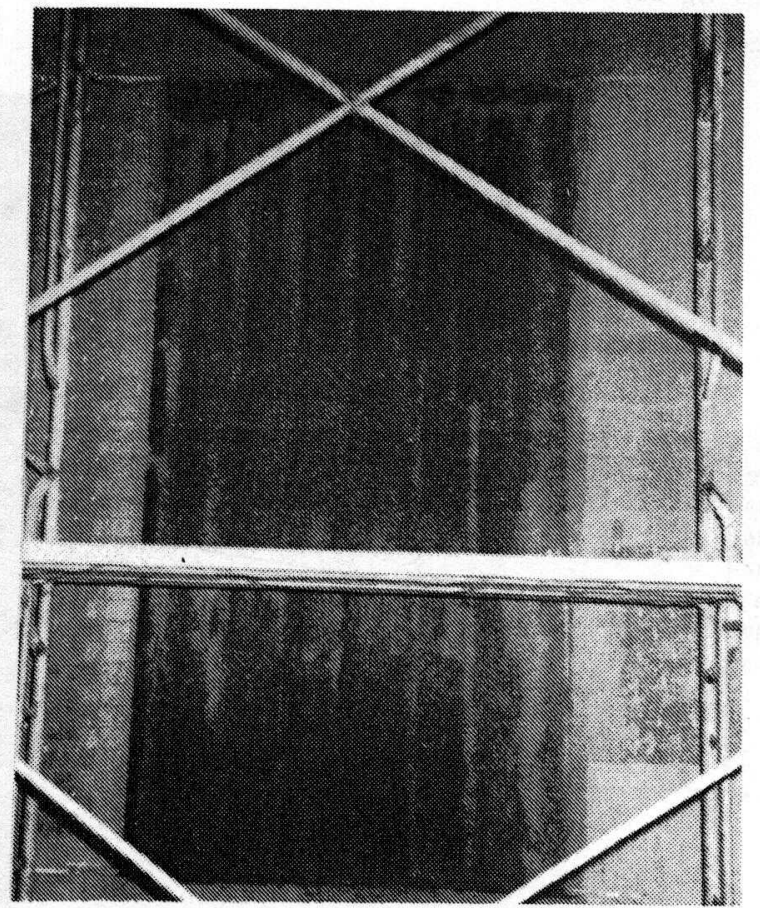

Fig.5 Appearance of laying undercoat by the robot 
sensor equipped on the trowel, the trowel starts to lay setting mortar on back tiles in the holding unit. By the way, the trowel can move to lay both upward and downward.

(d) Setting tiles

Setting method by the robot is especially invented to overcome the problem of open time which means the period during which tiles were still not as yet applied to set. The open time problem causes breakaway of tiles. Therefore, as soon as the robot complete to lay setting mortar on tiles, the robot sets tiles to undercoat of the wall. Each tile in the tile holding unit which was mounted setting mortar on back, is taken by the vacuum suction shocker equipped on the setting unit, and the tile is pushed to the wall and impacted by power vibrator to confirm the tile to the surface of the wall. This causes tiles set firmly to the wall, and there is less deviation and greater adhesive strength than conventional method. Thickness of setting mortar is $5 \mathrm{~mm}$, and finishing is $3 \mathrm{~mm}$ measured by stroke sensor equipped on the tile setting unit.

(2) Adhesive strength

The averaged strength after working 28 days past was obtained $15 \mathrm{Kgf} / \mathrm{cm}^{2}$, and even minimum strength was $11 \mathrm{kgf} / \mathrm{cm}^{2}$ both cross type and square type joint. Strength of setting by the robot was stronger than conventional and target $6 \mathrm{kgf} / \mathrm{cm}^{2}$. The test result is shown in Fig. 6.

(3) Evenness (Setting surface) accuracy

The robot achieved the greater evenness accuracy than target setting surface accuracy, that is, tolerance of $\pm 1 \mathrm{~mm}$ to a length of $2 \mathrm{~m}$. Fig. 7 shows the appearance of setting surface by the robot.

(4) Setting efficiency
Setting efficiency of the robot was verified $9 \mathrm{~m}^{2} /$ day better than conventional.

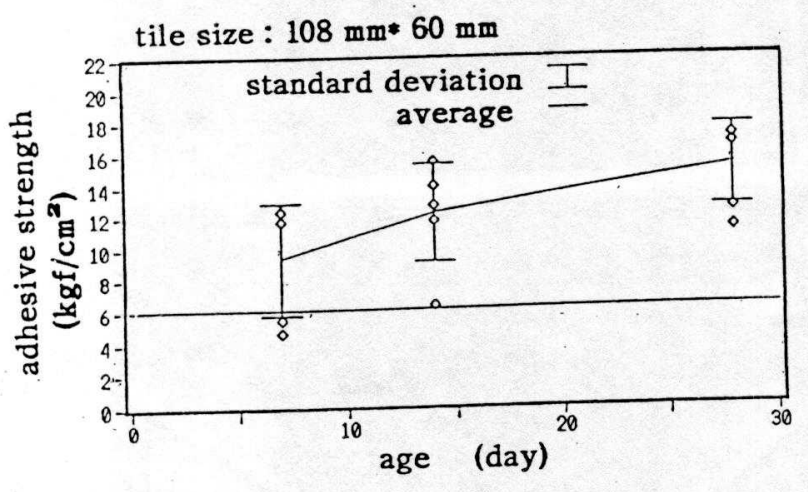

Fig. 6 Adhesive strength of setting tiles

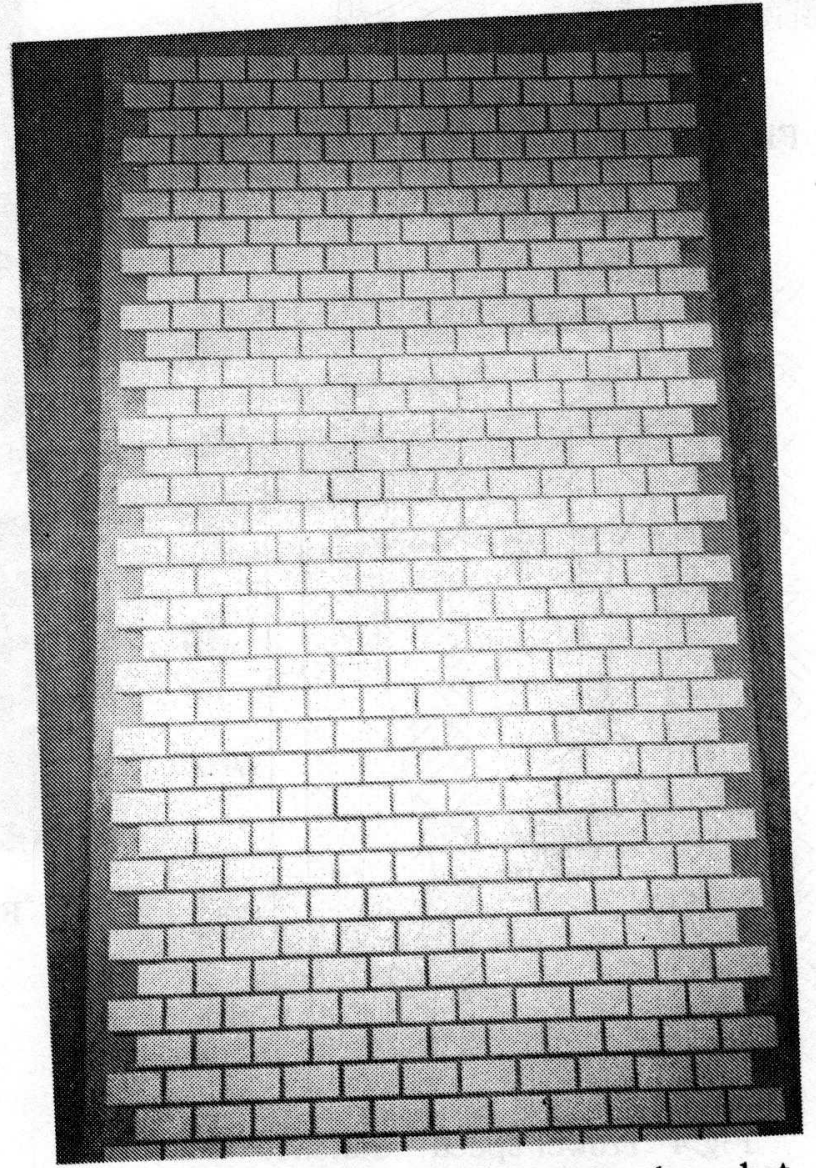

Fig. 7 Appearance of setting tiles by the robot 


\section{Results of working in site}

In December 1990, the tile setting robot was introduced into site working, Arakawa Ward Tokyo, to estimate and to prove practical use of the tile setting robot. The area of execution was about $10 \mathrm{~m}^{2}$ both laying undercoat and setting tiles (about 1300 pieces). The appearance of site working of the tile setting robot is shown in Fig. 8.

(1) Laying undercoat

But data of the adhesive strength laid by the robot was not obtained, the evenness accuracy was obtained equal to the indoor model wall test, that is, tolerance of $\pm 1 \mathrm{~mm}$ to a length of $2 \mathrm{~m}$. And laying efficiency of the robot was equal to the model wall test too. Fig. 9 shows the appearance of laying undercoat by the robot in site.

(2) Setting tiles

The average adhesive strength setting by the robot was obtained $12 \mathrm{Kgf} / \mathrm{cm}^{2}$, even minimum adhesive strength was $10 \mathrm{Kgf} / \mathrm{cm}^{2}$. And there is no break from the undercoat. Also finishing accuracy and setting efficiency of the robot was equal to the results of the model wall test. Therefore, The practical use of the robot was proved. Fig.10 shows the appearance of finishing surface by the robot in this test.

\section{Conclusion}

Komatsu Ltd., Japan Ceramic Tile Association, and Hazama corporation have been developing the tile setting robot which has two functions, one is to set tile for exterior wall of buildings, the other is to lay (mortaring and finishing) undercoat of buildings. And we tested the the robot on the indoor model wall and we applied the robot for construction work. We were satisfied with the results of indoor model wall test and application for field test. For adhesive strength, evenness accuracy, and efficiency of working by the robot in both laying undercoat and setting tiles were much greater than conventional.

As we prove the practical use of the tile setting robot both laying undercoat and setting tiles, we are going to further advance the robot which is to be more simply and easy to operate. And we would like to commercialize the tile setting robot near future.

\section{Reference}

(1) K.Matsumoto,K.Otsubo,S.Ishikawa "Development of Tile setting robot", The first symposium on Construction Robotics in Japan, June 1990, p357-366. 


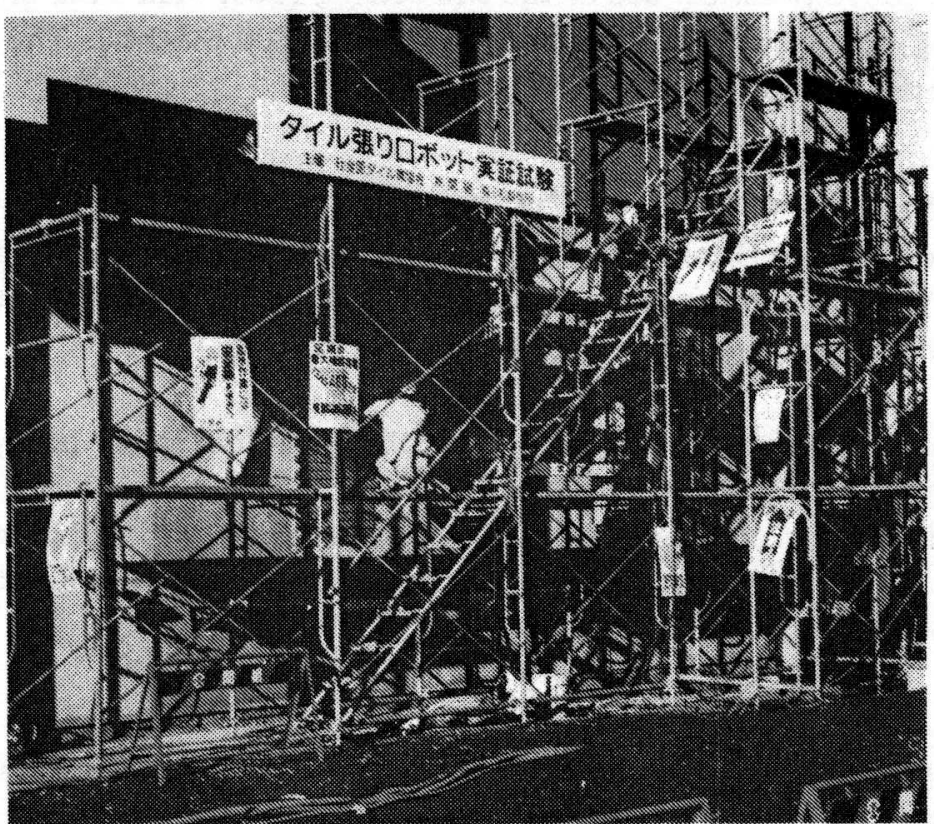

Fig. 8 Appearance of working robot in site

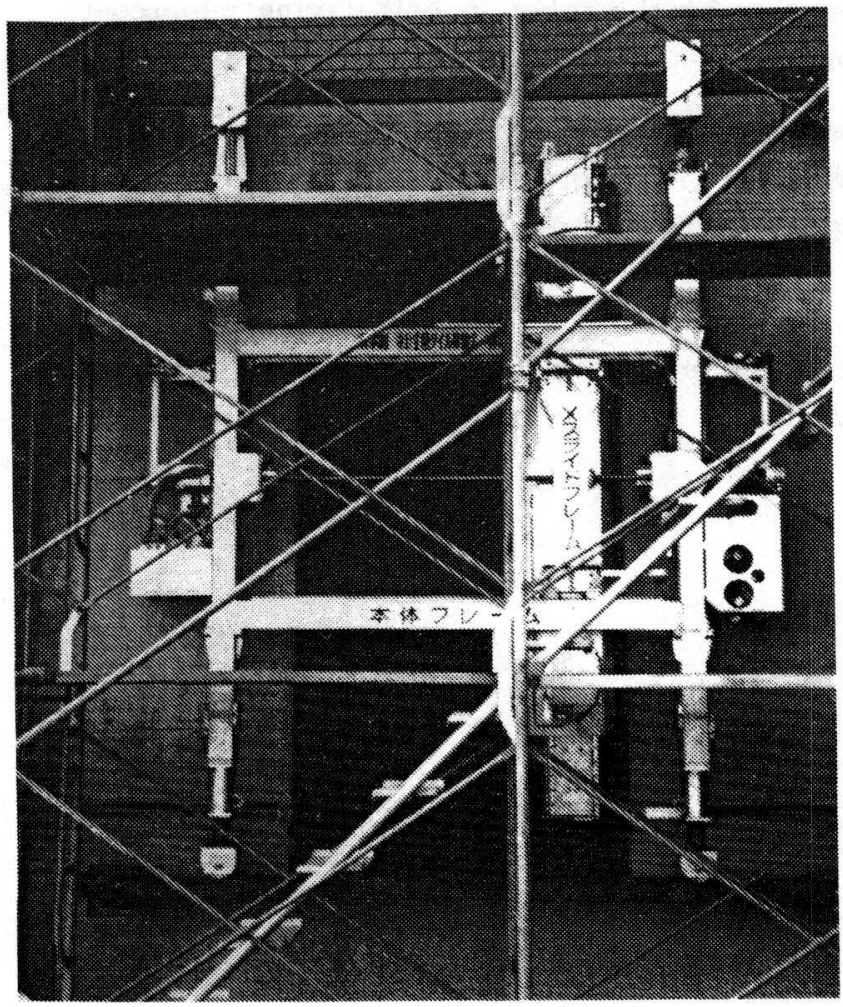

Fig.9 Appearance of laying undercoat

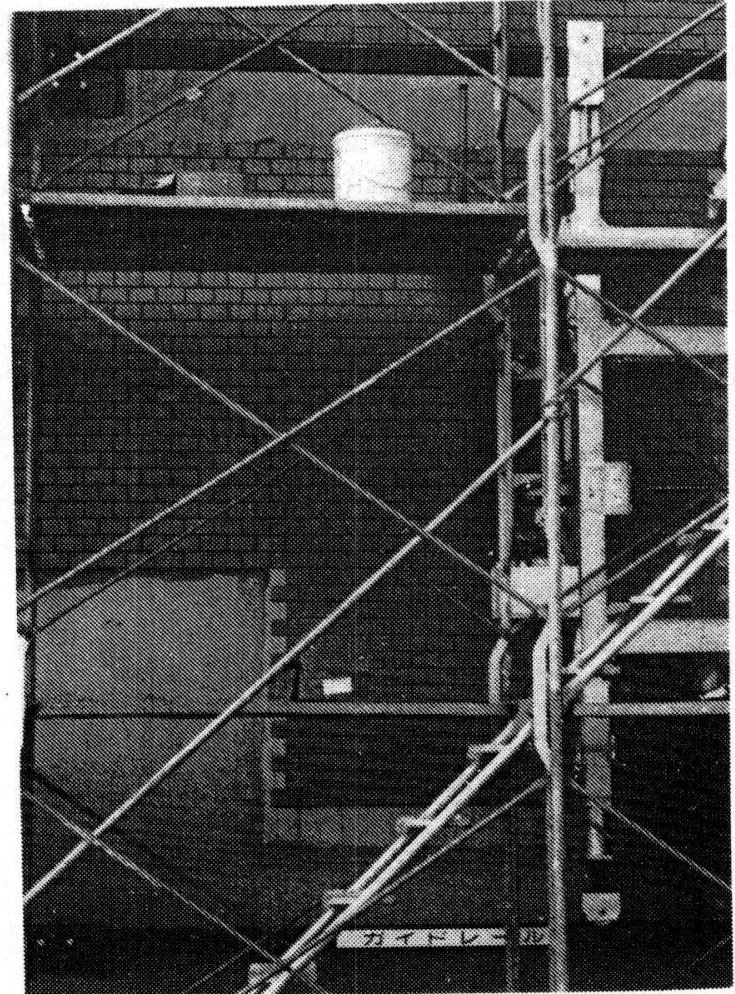

Fig.10 Appearance of setting tiles 